\title{
Cerebral correlates of psychotic symptoms in Alzheimer's disease
}

\begin{abstract}
Michael S Mega, Linda Lee, Ivo D Dinov, Fred Mishkin, Arthur W Toga, Jeffrey L Cummings
\end{abstract}

Department of Neurology UCLA School of Medicine, UCLA School of Medicine, Los Angeles, California, USA

M S Mega

L Lee

I D Dinov

A W Toga

J L Cummings

Laboratory of

Neuroimaging

M S Mega

L Lee

I D Dinov

A W Toga

Alzheimer's Disease ResearchCenter M S Mega

J L Cummings

Division of Nuclear

Medicine,

Harbor-UCLA

Medical Cmt

Torrance, California, USA

F S Mishkin

Correspondence to: Dr Michael S Mega, Laboratory of Neuro Imaging, Department of Neurology, UCLA School of Medicine, 710 Westwood Plaza, Los Angeles, CA 90095-1769, USA mega@loni.ucla.edu

Received 1 July 1999 and in revised form

16 November 1999

Accepted 14 December 1999

\begin{abstract}
Background-Psychotic symptoms are produced by distributed neuronal dysfunction. Abnormalities of reality testing and false inference implicate frontal lobe abnormalities.

Objectives-To identify the functional imaging profile of patients with Alzheimer's disease manifesting psychotic symptoms as measured by single photon emission computed tomography (SPECT).
\end{abstract}

Methods-Twenty patients

with

Alzheimer's disease who had SPECT and clinical evaluations were divided into two equal groups with similar mini mental status examination (MMSE), age, sex, and the range of behaviours documented by the neuropsychiatric inventory (NPI), except delusions and hallucinations. SPECT studies, registered to a probabilistic anatomical atlas, were normalised across the combined group mean intensity level, and subjected to a voxel by voxel subtraction of the non-psychotic minus psychotic groups. Subvolume thresholding (SVT) corrected random lobar noise to produce a three dimensional functional significance map.

Results-The significance map showed lower regional perfusion in the right and left dorsolateral frontal, left anterior cingulate, and left ventral striatal regions along with the left pulvinar and dorsolateral parietal cortex, in the psychotic versus non-psychotic group.

Conclusion-Patients with Alzheimer's disease who manifest psychosis may have disproportionate dysfunction of frontal lobes and related subcortical and parietal structures.

(F Neurol Neurosurg Psychiatry 2000;69:167-171)

Keywords: brain mapping; neuropsychiatric inventory; HMPAO-SPECT; behaviour

Psychosis is common in Alzheimer's disease: delusions affect $10 \%$ to $73 \%$ of patients with Alzheimer's disease ${ }^{1-10}$ and hallucinations 3\% to $49 \% .^{5-13}$ Delusions are most prevalent in women ${ }^{9}$ and during the middle phase of the illness. ${ }^{2514}$ Psychotic patients with Alzheimer's disease exhibit an accelerated cognitive decline. ${ }^{15-17}$ Visual hallucinations in Alzheimer's disease are more common than auditory hallucinations and, if accompanied by a fluctuating course or extrapyramidal signs, may suggest dementia with Lewy bodies. ${ }^{18}$ Although delusions increase from the mild to moderate stage of Alzheimer's disease, hallucinations decline in more severely impaired patients. ${ }^{14}$ The high prevalence of psychotic symptoms allows mapping of regional defects associated with delusions and hallucinations. Psychotic symptoms are correlated with metabolic and perfusion abnormalities in frontal, ${ }^{19-21}$ temporal, ${ }^{22-24}$ and parietal cortex ${ }^{20}$ in Alzheimer's disease. Exploring the neural basis of psychotic symptoms in dementia requires control over prevalent concomitant behaviours, a methodological refinement not pursued in previous studies.

Psychotic symptoms do not occur in isolation. Agitation, anxiety, and irritability may be associated with increasing psychosis depending on the stage of dementia in Alzheimer's disease. ${ }^{14}$ Aarsland et $a l^{25}$ reported that psychotic behaviour accounted for nearly $25 \%$ of the variance in measures of aggression in 75 patients with Alzheimer's disease. Controlling for accompanying behaviours is necessary in functional imaging studies that attempt to map the neuronal basis of symptoms such as psychosis in any disease population. The neuropsychiatric inventory (NPI) is a care giver based instrument ${ }^{26}$ that evaluates 10 troublesome behaviours in patients with dementia and provides the means for controlling for a wide range of behaviours in a functional imaging subtraction paradigm. We used the NPI to isolate psychotic symptoms, while controlling for other abnormal behaviours, in an Alzheimer's disease population undergoing technetium$99 \mathrm{~m} \mathrm{~d}, 1$, hexamethylpropeleneamine oxime ( ${ }^{99 \mathrm{~m}}$ Tc-HMPAO) single photon emission computed tomography (SPECT) and clinical evaluation.

\section{Methods}

PATIENTS

Starting from a pool of 280 patients with Alzheimer's disease presenting to the University of California, Los Angeles (UCLA) Alzheimer's Disease Centre, 20 outpatients were selected who met all clinical criteria described below and enabled equal group means across demographic and behavioural domains of the psychotic and non-psychotic groups. All patients met National Institute of Neurological and Communicative Disorders and Stroke/Alzheimer's Disease and Related Disorders Association (NINCDS/ADRDA) criteria for probable or possible Alzheimer's disease. ${ }^{27}$ In addition, all patients had acquired persistent decline involving at least three of the following domains: language, memory, 
visuospatial skills, cognition (calculation, abstraction, judgment, etc), and emotion or personality. ${ }^{28}$ Severity of cognitive deficit was measured in all patients using the mini mental state examination (MMSE). ${ }^{29}$

\section{BEHAVIOURAL ASSESSMENT}

Family members living with the patient were interviewed with the NPI after procedures previously described ${ }^{26}$ in which screening questions for each behaviour were first posed. The care giver was asked if the behaviour represented a change from that exhibited by the patient before the onset of the dementia and if it was present during the past month. If a positive response was obtained then the behavioural domain was explored with scripted questions focusing on specific features of the behavioural disturbance. The care givers were then asked to rate the behaviours; scores from 1-4 were obtained for the frequency and 1-3 for the severity of each behaviour (a composite score for each domain was the product of the frequency and severity subscores; maximum=12). The 10 domains assessed using the NPI are delusions, hallucinations, agitation, depression, anxiety, euphoria, apathy, disinhibition, irritability, and abnormal motor output. The MMSE was administered at the same time as the NPI.

SPECT SCANNING

For all patients, an intravenous line was placed and 15 minutes were allowed to pass for patients to regain a quiet comfortable state before the intravenous administration of 30 $\mathrm{mCi}^{99 \mathrm{~m}} \mathrm{Tc}$ labelled HMPAO (Ceretec; Amersham, Arlington Heights, IL, USA). Room lights were dimmed and the rooms kept quiet to minimise visual and auditory stimuli during the 15 minute brain uptake phase after injection. About 1 hour after injection, during which time washout of the tracer from the brain had occurred, SPECT images of the brain were obtained using a Picker 3000XP SPECT scanner (Picker International, Inc, Cleveland, OH, USA) with low energy ultrahigh resolution fan beam collimators. Images were reconstructed by filtered back projection using a low pass filter, eighth order, with a spatial frequency cut off of 0.23 to 0.25 cycles/ pixel. Transverse, sagittal, and coronal planes with a $128 \times 128$ matrix were generated. Pixel sizes were nominally $3.56 \times 3.56 \mathrm{~mm}$. Resolution of the system was about $6 \mathrm{~mm}$ full width at half maximum (FWHM).

IMAGE PROCESSING

Spatial alignment of all 20 SPECT datasets was accomplished via 12 parameter affine registration. ${ }^{30}$ All datasets were first aligned and averaged to a random SPECT target to obtain an average "composite SPECT" which in turn was aligned to the International Consortium of Human Brain Mapping (ICBM) probabilistic atlas. ${ }^{31}$ To minimise resampling of data, the two registration fields above were concatenated and applied to each SPECT dataset. The relative perfusion scans of each patient then underwent linear intensity nor- malisation, on a voxel by voxel basis, to the global mean intensity value of all 20 patients, thus equalising the mean intensities across all datasets. This normalisation step did not alter the intersubject data variance or the mean intergroup differences.

Once all normalised datasets were in the common ICBM atlas space, a voxel by voxel subtraction was conducted between the psychotic and non-psychotic groups. Subvolume thresholding (SVT) was used to create a statistical map of these subtraction results according to methods previously detailed. ${ }^{32}$ Briefly, SVT utilises the probabilistic anatomical partitioning of the ICBM atlas (regions include the frontal, parietal, temporal, insular, and occipital cortex, along with the putamen, caudate, thalamus, and cerebellum) to model the different regions as separate stationary random fields thereby accommodating nonuniform global brain activity. This novel approach is particularly well suited for the assessment of functional imaging studies in Alzheimer's disease as parietal and temporal regions may have different means and variances, across subjects, than frontal or subcortical regions given the pathological distribution of the disease. ${ }^{33}{ }^{34}$ Ignoring these potential differences by modelling the entire dataset as a stationary random field, done by many other functional assessments, will obliterate disease specific variability.

After the location of voxels within a region of interest (ROI) has been assigned a $\mathrm{Z}$ score value, a significance level must be determined for voxels above a Z score threshold. The SVT local search within globally significant regions derived from the between group subtraction is corrected for multiple voxelwise testing to control for type I errors in assessing significance. For each of the voxels selected by SVT a Bonferroni correction is conducted by dividing the significance level associated with the $\mathrm{Z}$ score by the number of voxels constituting a single search (this voxel number is equal to the size of the FWHM of the scanner- $6 \mathrm{~mm}$ )

\section{Results}

Table 1 shows the demographic and behavioural profile of the two groups. The demographic profile and MMSE mean score of the patients in this study were similar to the larger

Table 1 Demographic and behavioural profile for the psychotic and non-psychotic groups as reflected by the composite (frequency $\times$ severity) scores on the 10 behavioural domains captured by the neuropsychiatric inventory (NPI)

\begin{tabular}{llll}
\hline & $\begin{array}{l}\text { Psychotic mean } \\
\text { (SEM) }\end{array}$ & $\begin{array}{l}\text { Non-psychotic } \\
\text { mean (SEM) }\end{array}$ & p Value \\
\hline Sex & $0 \mathrm{M} / 10 \mathrm{~F}$ & $1 \mathrm{M} / 9 \mathrm{~F}$ & \\
MMSE & $14.30(2.31)$ & $17.40(2.07)$ & 0.37 \\
Education & $11.50(2.39)$ & $12.89(2.54)$ & 0.49 \\
Delusions & $4.50(0.50)$ & $0.00(0.49)$ & 0.00 \\
Hallucinations & $1.30(0.14)$ & $0.00(0.14)$ & 0.01 \\
Agitation & $2.50(0.36)$ & $1.90(0.28)$ & 0.54 \\
Depression & $1.20(0.38)$ & $1.30(0.39)$ & 0.90 \\
Anxiety & $2.70(0.31)$ & $1.30(0.30)$ & 0.28 \\
Euphoria & $0.20(0.06)$ & $0.40(0.06)$ & 0.56 \\
Apathy & $4.40(0.58)$ & $3.20(0.45)$ & 0.37 \\
Disinhibition & $1.70(0.61)$ & $1.20(0.18)$ & 0.65 \\
Irritability & $2.30(0.61)$ & $2.00(0.26)$ & 0.81 \\
Aberrant motor & $1.90(1.20)$ & $3.20(0.31)$ & 0.44 \\
\hline
\end{tabular}




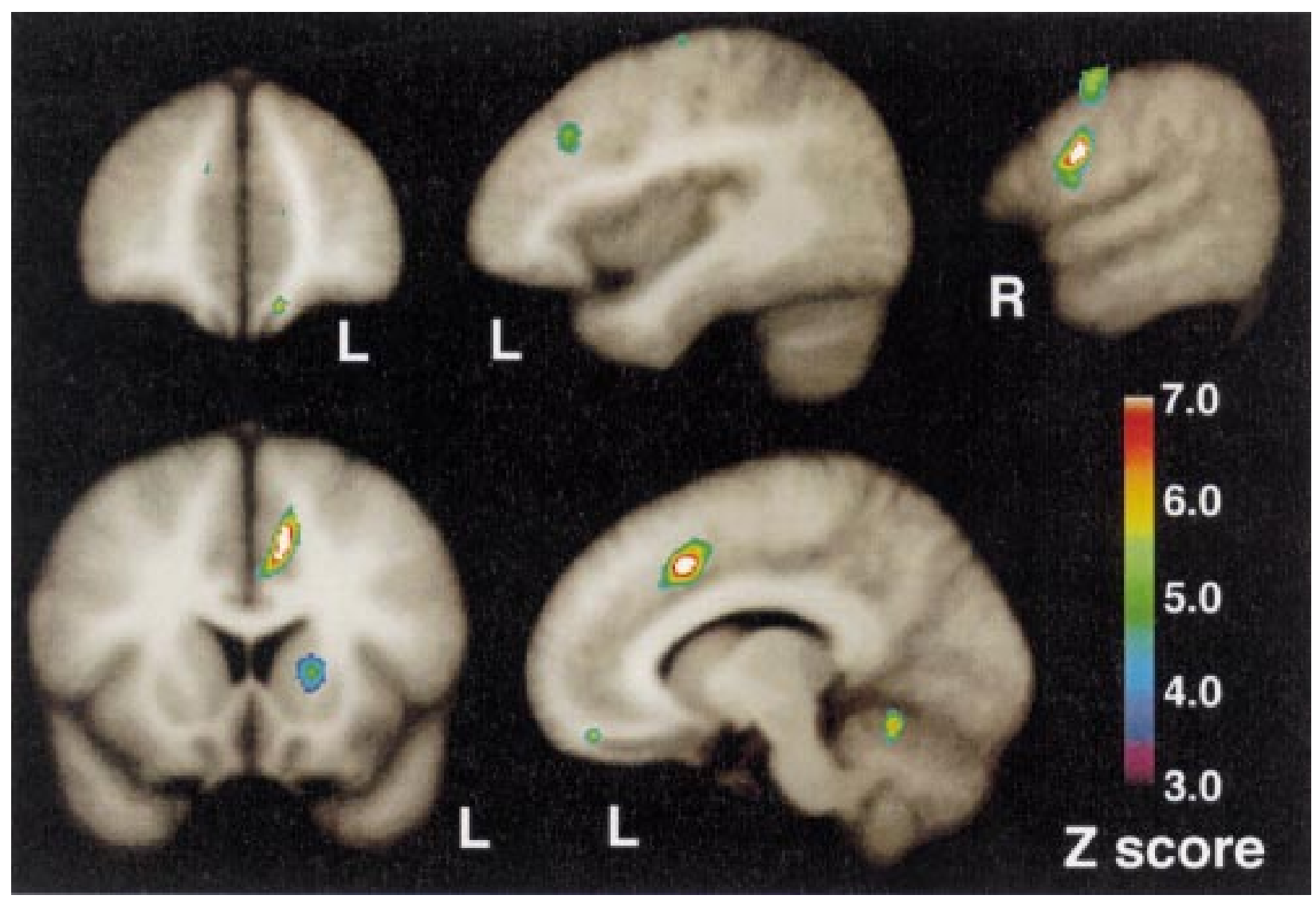

Statistical map of the regional, Bonferroni corrected, analysis of the non-psychotic $(n=10)$ minus psychotic $(n=10)$ patients with Alzheimer's disease. The map shows Talairach atlas location of the hypoperfused regions derived from normalised ${ }^{99 m}$ Tc-HMPAO SPECT, associated with psychotic symptoms.

patient pool except for the overrepresentation of women in our two groups. Delusions $(p<0.001)$ and hallucinations $(p<0.01)$ were the only behaviours that were significantly different between the two groups. All psychotic patients had delusions and half had hallucinations. Trends for lower MMSE ( $p=0.37)$, greater agitation $(\mathrm{p}=0.54)$, and anxiety $(p=0.28)$, with lower aberrant motor behaviour $(\mathrm{p}=0.44)$, were present in the psychotic group compared with the non-psychotic group. The figure and table 2 show the Talairach atlas ${ }^{35}$ location of the peak significance for regions with significantly lower perfusion in the 10 patients with Alzheimer's disease who had psy-

Table 2 The Talairach atlas ${ }^{35}$ location of brain regions'peak, Bonferroni corrected, significance for the statistical map shown in the figure.

\begin{tabular}{lll}
\hline Structure (Brodmann area) & $\begin{array}{l}\text { Talairach coordinates } \\
(x, y, z)\end{array}$ & Peak $z$ score $(p$ Value) \\
\hline Left medial orbital frontal (BA 11) & $-11,46,-14$ & $6.2(<0.0001)$ \\
Left anterior cingulate (BA 32) & $-9,14,46$ & $8.5(<0.0001)$ \\
Left dorsolateral frontal (BA 4) & $-58,-5,29$ & $6.5(<0.0001)$ \\
Left dorsolateral frontal (BA 8) & $-39,23,37$ & $5.4(<0.0001)$ \\
Left dorsolateral parietal (BA 40) & $-57,-52,28$ & $5.2(<0.0001)$ \\
Left pulvinar & $-12,-26,9$ & $4.5(<0.0001)$ \\
Left ventral striatum & $-16,13,8$ & $5.2(<0.0001)$ \\
Left cerebellum & $-11,-59,-13$ & $7.4(<0.0001)$ \\
Right dorsolateral frontal (BA 4) & $53,-7,49$ & $6.6(<0.0001)$ \\
Right dorsolateral frontal (BA 6) & $59,-2,28$ & $9.3(<0.0001)$ \\
Right medial frontal (BA 8) & $11,27,42$ & $4.7(<0.0001)$
\end{tabular}

These locations were derived from normalised brain perfusion, as measured by ${ }^{99 \mathrm{~m}} \mathrm{Tc}-\mathrm{HMPAO}$ SPECT. The map reflects regions with significantly lower perfusion in 10 patients with Alzheimer's disease who had significantly more delusions and hallucinations compared with 10 patients with Alzheimer's disease who were matched demographically and across all other behaviours measured by the NPI. chotic symptoms compared with the 10 non-psychotic patients with Alzheimer's disease as reported by care givers. The regions showing significantly lower perfusion in the psychotic group included the left and right prefrontal, left striatum, and left parietal cortex.

\section{Discussion}

Functional imaging is the best tool for exploring the neuronal basis of neuropsychiatric disorders in life. Functional in vivo dissection of neural systems is only possible with metabolic or perfusion imaging. Psychosis is a complex behavioural disorder that does not manifest from a single brain defect. Disordered reality testing and abnormal inferential thinking are fundamental to psychosis. Such a disorder implicates executive and internal monitoring defects, and abnormal assessment of the emotional relevance of stimuli. We found significant hypoperfusion in the dorsolateral frontal cortex bilaterally, the left anterior cingulate, ventral striatum, pulvinar, and dorsolateral parietal cortex in psychotic patients with Alzheimer's disease. Both right motor (BA 4, 6, and 8), left prefrontal (BA 11, 8, and 4), and cingulate regions were hypoperfused supporting a defect in motor planning and cognitive executive function as well as the cingulate attentional system.

The left dorsolateral frontal lobe integrates language based executive function ${ }^{36-39}$ whereas the right seems to subserve internal monitoring 
of veridical choice. ${ }^{40} 41$ The anterior cingulate coordinates executive and self monitoring systems to simultaneously operate on modality specific sensory and association networks ${ }^{42-44}$ while coordinating the posterior parietal and dorsolateral frontal attentional network. ${ }^{43}$ Outflow from the anterior cingulate is directed to the ventral striatum, ${ }^{45}$ also termed the limbic striatum. ${ }^{46}$ This medial frontal subcortical circuit may coordinate the integration of emotionally relevant tone with executive processing; dysfunction could result in emotionally charged aberrant beliefs. Lesions of the left orbitofrontal cortex produce spontaneous confabulation. ${ }^{47}{ }^{48}$ Dysfunction of these reciprocally connected frontal networks is here associated with the occurrence of psychosis in Alzheimer's disease.

No single brain location will be the source of psychotic symptoms in Alzheimer's disease but the medial and dorsolateral frontal cortical networks, in conjunction with the anterior cingulate limbic circuits, subserve many of the functions that seem to disintegrate with increasing psychotic symptoms. These regions have been implicated in the current study. This study has a small sample size selected from nearly 300 patients in an effort to produce similar demographic groups and control concomitant abnormal behaviours associated with psychotic systems. The drawback of such a sample purification is the loss of representation of a general Alzheimer's disease population as reflected by the low representation of men in this study. Yet the purification of the groups was needed to isolate and balance behaviours. Perfect balance was not achieved, however, given the trend for lower MMSE, higher agitation, and anxiety, with lower aberrant motor behaviour in the psychotic group. Future studies should determine if the neuronal basis of psychosis is similar across diseases and what baseline functional imaging patterns might predict treatment response.

Support for this work was provided by an NIA career development award (K08AG100784) to MSM; an NIA Alzheimer's Disease Research Center grant (P50 AG16570); an Alzheimer's Disease Research Center of California grant; the Sidell-Kagan Foundation, and the Human Brain Project (NIMH/NIDA: Foundation, and the Human Brain Project (NIMH/NIDA:
P20MH/DA 52176, NSF (BIR9322434), NCRR (RR05956).

1 Birkett DP. The psychiatric differentiation of senility and arteriosclerosis. Br F Psychiatry 1972;120:321-5.

2 Cummings JL, Miller B, Hill MA, et al. Neuropsychiatric aspects of multi-infarct dementia and dementia of the aspects of multi-infarct dementia and dem

3 Merriam AE, Aronson MK, Gaston P, et al. The psychiatric symptoms of Alzheimer's disease. I Am Geriatr Soc symptoms of

4 Kumar A, Koss E, Metzler D, et al. Behavioral symptomatology in dementia of the Alzheimer type. Alzheimer Dis Assoc Disord 1988;2:363-5.

5 Wragg RE, Jeste DV. Overview of depression and psychosis in Alzheimer's disease. Am f Psychiatry 1989;45:577-87.

6 Copper JK, Mungas D, Weiler PG. Relation of cognitive status and abnormal behaviors in Alzheimer's disease. $f \mathrm{Am}$ Geriatr Soc 1990;38:867-70

7 Cohen D, Eisdorfer C, Gorelick P, et al. Psychopathology associated with Alzheimer's disease and related disorders. $f$ Gerontol 1993;48:M255-60.

8 Gormley N, Rizwan MR. Prevalence and clinical correlates of psychotic symptoms in Alzheimer's disease. International fournal of Geriatric Psychiatry 1998;13:410-14

9 Hirono N, Mori E, Yasuda M, et al. Factors associated with psychotic symptoms in Alzheimer's disease. $\mathcal{F}$ Neurol psychotic symptoms in Alzheimer's
Neurosurg Psychiatry 1998;64:648-52.

10 Migliorelli R, Petracca G, Teson A, et al. Neuropsychiatric and neuropsychological correlates of delusions in Alzheimer's disease. Psychol Med 1995;25:505-13.
11 Berrios GE, Brook P. Visual hallucinations and sensory delusions in the elderly. Br f Psychiatry 1984;144: 662-4.

12 Sinha D, Zemlan FP, Nelson S, et al. A new scale for assessing behavioral agitation in dementia. Psychiatry Res 1992;41:73-88.

13 Gilley DW, Whalen ME, Wilson RS, et al. Hallucinations and associated factors in Alzheimer's disease. F Neuropsychiatry Clin Neurosci 1991;3:371-6.

14 Mega MS, Cummings JL, Fiorello T, et al. The spectrum of behavioral changes in Alzheimer's disease. Neurology 1996; 46:130-5.

15 Drevets WC, Rubin EH. Psychotic symptoms and the longitudinal course of senile dementia of the Alzheimer type. Biol Psychiatry 1989;25:39-48.

16 Frisoni GB, Rozzini L, Gozzetti A, et al. Behavioral syndromes in Alzheimer's disease: description and correlates. Dement Geriatr Cogn Disord 1999;10:130-8.

17 Levy ML, Cummings JL, Fairbanks LA, et al. Longitudinal assessment of symptoms of depression, agitation, and psychosis in 181 patients with Alzheimer's disease. Am f Psychiatry 1996;153:1438-43.

18 McKeith IG, Galasko D, Kosaka K, et al. Clinical and pathological diagnosis of dementia with Lewy bodies (DLB): report of the CDLB international workshop. Neurology 1996;46:1113-24.

19 Grady CL, Haxby JV, Schapiro MB, et al. Subgroups in dementia of the Alzheimer's type identified using positron emission tomography. $f$ Neuropsychiatry Clin Neurosci 1990;2:373-84

20 Kotrla KJ, Chacko RC, Harper RG, et al. SPECT findings on psychosis in Alzheimer's disease. Am f Psychiatry 1995; 152:1470-5

21 Sultzer DL, Mahler ME, Mandelkern MA, et al. The relationship between psychiatric symptoms and regional cortical metabolism in Alzheimer's disease. 7 Neuropsychiatry Clin Neurosci 1995;7:476-84.

22 Starkstein SE, Vazquez S, Petracca G, et al. A SPECT study of delusions in Alzheimer's disease. Neurology 1994;44: 2055-9.

23 Mentis MJ, Weinstein EA, Horwitz B, et al. Abnormal glucose metabolism in the delusional misidentification syndromes: a positron tomography study in Alzheimer's disease. Biol Psychiatry 1995;38:438-49.

24 Gustafson L, Risberg J. Regional cerebral blood flow related to psychiatric symptoms in dementia with onset in the presenile period. Acta Psychiatr Scand 1974;50: 516-38.

25 Aarsland D, Cummings JL, Yenner G, et al. Relationship of aggressive behavior to other neuropsychiatric symptoms in patients with Alzheimer's disease. Am $\mathcal{F}$ Psychiatry 1996; 153:243-7.

26 Cummings JL, Mega MS, Gray K, Rosenberg-Thompson S, Carusi DA, Gornbein J. The neuropsychiatric inventory: comprehensive assessment of psychopathology in demencomprehensive assessment of psych
tia. Neurology 1994;44:2308-2314.

27 McKhann G, Drachman D, Folstein M, Katzman R, Price D, Stadlan EM. Clinical diagnosis of Alzheimer's disease: report of the NINCDS-ADRDA Work Group, Department of Health and Human Services Task Force on Alzheimer's Disease. Neurology 1984;34: 939-944.

28 Cummings JL, Benson DF, LoVerme S. Reversible dementia: illustrative cases, definition, and review. $7 A M A$ 1980;243:2434-2439.

29 Folstein MF, Folstein SE, McHugh PR. "Mini-mental state": A practical method for grading the mental state of
patients for the clinician. 7 Psychiatry Res $1975 ; 12: 189-$ 198.

30 Woods RP, Grafton ST, Watson JDG, Sicotte NL, Mazziotta JC. Automated image registration: II. Intersubject validation of linear and nonlinear models. F Comput Assist Tomogr 1998;22:153-165.

31 Evans AC, Collins DL, Holmes CJ. Automated 3D regional MRI segmentation and statistical probabilistic anatomical maps. In: Toga AW, Mazziotta JC, ed. Human Brain Mapping: the methods. New York: Academic Press, 1996: 123-130.

32 Dinov ID, Mega MS, Thompson PM, et al. Analyzing functional brain images in a probabilistic atlas: a validation of Sub-volume thresholding. F Comput Assist Tomogr 1999; 2000;34:128-39.

33 Price JL, Davis PB, Morris JC, White DL. The distribution of tangles, plaques and related immunohistochemical markers in healthy aging and Alzheimer's disease. Neurobiol Aging 1991;12:295-312.

34 Braak H, Braak E. Neuropathological staging of Alzheimerrelated changes. Acta Neuropathol 1991;82:239-259.

35 Talairach J, Tournoux P. Principe et technique des etudes anatomiques. In: Rayport M, ed. Co-Planar Stereotaxic Atlas of the Human Brain - 3-Dimensional Proportional System: An Approach to Cerebral Imaging. New York: Thieme Medical Publishers, Inc, 1988:3-9.

36 Alexander MP, Benson DF, Stuss DT. Frontal lobes and language. Brain and Language 1989;37:656-691.

37 Benton AL. Differential behavioral effects in frontal lobe disease. Neuropsychologia 1968;6:53-60.

38 Mega MS, Cummings JL. Frontal subcortical circuits and neuropsychiatric disorders. $\mathcal{F}$ Neuropsychiatry Clin Neurosci 1994;6:358-370.

39 Shaywitz BA, Pugh KR, Constable RT, et al. Localization of semantic processing using functional magnetic resonance imaging. Human Brain Mapping 1995;2:149-158. 
40 Fletcher PC, Firth CD, Grasby PM, Shallice T, Frackowiak RSJ, Dolan RJ. Brain systems for encoding and retrieval of auditory-verbal memory. An in vivo study in humans. Brain (1)

41 Shallice T, Fletcher P, Firth CD, Grasby P, Frackowiak RSJ, Dolan RJ. Brain regions associated with acquisition and retrieval of verbal episodic memory. Nature 1994;368:633635

42 Mega MS, Cummings JL. The cingulate and cingulate syndromes. In: Trimble MR, Cummings JL, ed. Contemporary Behavioral Neurology. Boston: Butterworth-Heinemann, 1997:189-214

43 Morecraft RJ, Geula C, Mesulam M-M. Architecture of connectivity within a cingulfronto-parietal neurocognitive network. Arch Neurol 1993;50:279-284.
44 Vogt BA, Finch DM, Olson CR. Functional heterogeneity in cingulate cortex: the anterior executive and posterio

45 Selemon LD, Goldman-Rakic PS. Longitudinal Topography and interdigitation of corticostriatal projections in the rhesus monkey. I Neurosci 1985;5:776-794.

46 Heimer L. The olfactory cortex and the ventral striatum. In: Livingston KE, Hornykiewisz O, eds. Limbic mechanisms. New York: Plenum Press, 1978:95-187.

47 Schnider A, von Däniken C, Gutbrod K. The mechanism of spontaneous and provoked confabulations. Brain 1996; 119:1365-1375.

48 Schnider A, von Däniken C, Gutbrod K. Disorientation in amnesia: a confusion of memory traces. Brain 1996;119: 1627-32.

\section{NEUROLOGICAL STAMP}

\section{Emil Theodore Kocher (1841-1917)}

Theodore Kocher was Professor of Surgery at Berne for almost half a century. His experimental studies which included those on coagulation of blood, function of the brain and spinal cord, investigation of intracranial pressure, and

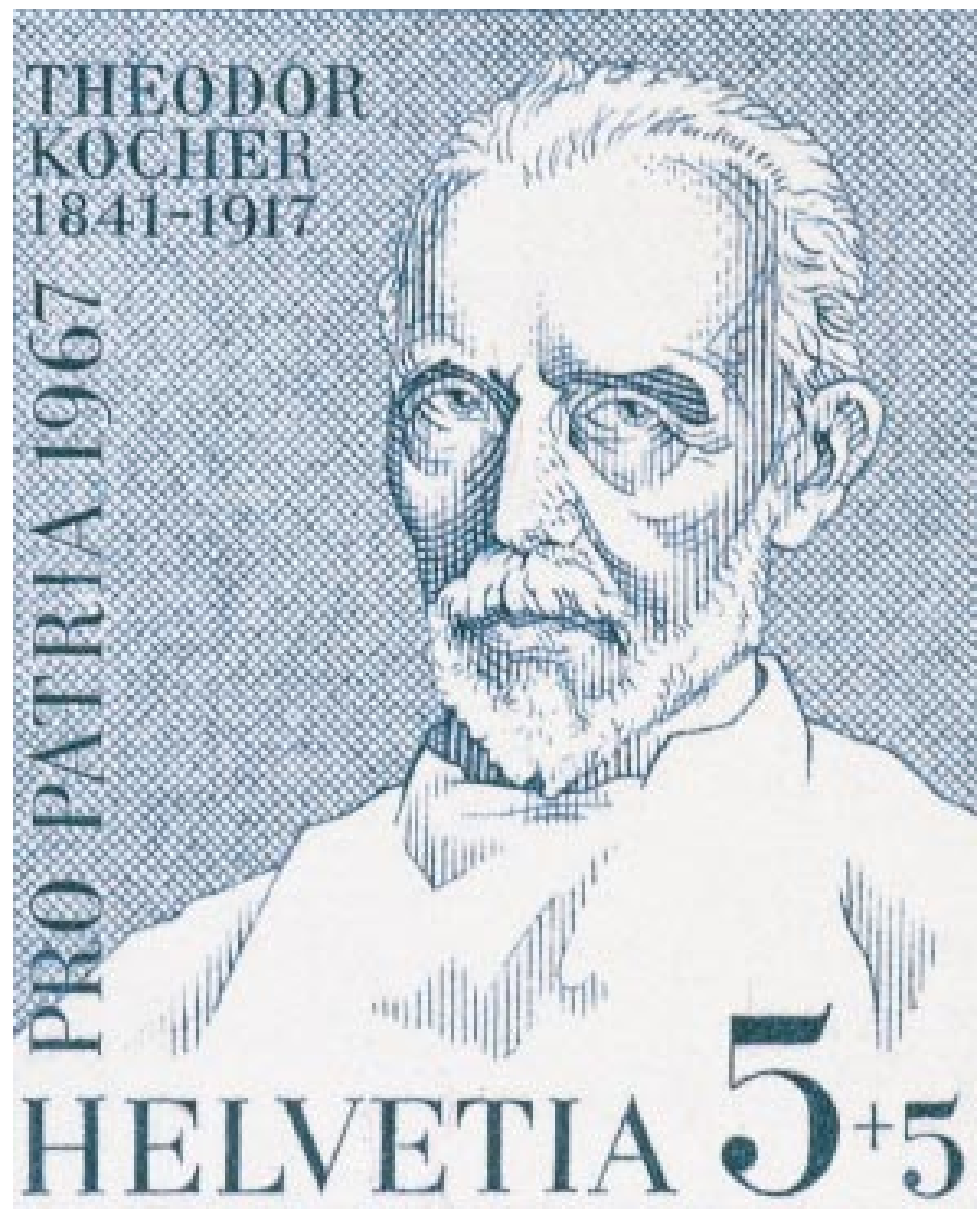

bullet wounds and his contributions to general surgery were overshadowed by his pioneer work on the thyroid gland. He became the first surgeon to receive the Nobel Prize in Physiology or Medicine. The Nobel Committee cited Kocher for his "work on the physiology, pathology and surgery of the thyroid gland". Goitre was especially common in Switzerland. In 1883 he found that around one third of his patients who had undergone thyroidectomy developed postoperative myxoedema and the associated idiocy and associated symptoms were indistinguishable from cretinism. He showed that these tragedies could be prevented by not removing the whole of the thyroid gland. By 1898 Kocher reported a series of 600 thyroidectomies with only a single death. $\mathrm{He}$ emphasised avoidance of injury to the recurrent laryngeal nerves, which could lead to changes in the voice and tracheal obstruction especially if both recurrent nerves were injured. At the end of his career Kocher had performed more than 5000 thyroldectomies for goitre with a very low mortality of $1 \%$. Calm, cool, imperturbable, and deliberate, Kocher was complete master of all surgical situations. For many years his clinic was a mecca for visiting surgeons from all parts of the world. Harvey Cushing as a young man "found Horsley pre-occupied and everyone else in England on their holidays". He left England and went to Berne to work with Kocher, who had neurological interests. Cushing's first impression was recorded in his diary for 1 November 1900. He commented on his "detailed technique, tedious operating, absolute hemostasis". Of interest, Horsley's first work was on myxoedema, for which he suggested implantation of normal thyroid tissue. In 1967 the Swiss honoured Kocher philatelically on the 50th anniversary of his death (Stanley Gibbons 746, Scott B365).

LF HAAS 